\title{
APLICAÇÃO DE UMA FONTE ALTERNATIVA DE ENERGIA TERMELÉTRICA A GÁS NATURAL VISANDO REDUZIR O CUSTO COM ENERGIA ELÉTRICA EM UM EDIFÍCIO COMERCIAL
}

\author{
A. A. OCHOA ${ }^{1 *}$, H. DINIZ ${ }^{2}$, W. SANTANA ${ }^{2}$, P. SILVA ${ }^{2}$ e L. OCHOA ${ }^{3}$ \\ ${ }^{1}$ Instituto Federal de Tecnologia de Pernambuco \\ ${ }^{2}$ Universidade Salgado de Oliveira Universo \\ ${ }^{3}$ Universidade Federal de Alagoas \\ ochoaalvaro@recife.ifpe.edu.br
}

Artigo submetido em setembro/2014 e aceito em fevereiro/2015

DOI: $10.15628 /$ holos.2015.2362

\section{RESUMO}

Este trabalho tem como objetivo reduzir o consumo de energia elétrica de um Edifício Comercial, localizado em Recife- PE, através da utilização de uma unidade termelétrica (grupo gerador) que opera com gás natural. A unidade termelétrica servirá como uma fonte alternativa de energia na matriz energética do empreendimento. $\mathrm{O}$ custo de energia elétrica representa um impacto considerável nos custos operacionais do edifício. A análise quantitativa dos dados de consumo de energia do Edifício Comercial estudado, associado ao conhecimento do sistema tarifário hora-sazonal foi fundamental para determinar a viabilidade do projeto. Além disto, foi verificada a viabilidade econômica do projeto, tendo como base dois parâmetros financeiros de tempo de retorno (payback) e critério do valor presente líquido (CVPL). Os resultados permitiram demonstrar, que realmente é tecnicamente e economicamente viável a aplicação do investimento neste projeto, obtendo uma redução de consumo de energia elétrica de aproximadamente $20 \%$ a. m., o equivalente a $\mathrm{R} \$ 295.000,00$ menos do valor pago mensalmente. Outro ganho é que o Edifício Comercial diversificou suas fontes de energia elétrica e assim permanecerá com alimentação elétrica quando houver interrupção no fornecimento da rede pública.

PALAVRAS-CHAVE: Análise financeira, Termelétrica, Redução de Consumo de Energia.

\section{APPLICATION OF AN ALTERNATIVE SOURCE OF NATURAL GAS POWER PLANTS AIMING TO REDUCE THE COST OF ELECTRICITY IN A COMMERCIAL BUILDING}

\begin{abstract}
This work aims to study and verification of an enterprise, through financial methods to reduce the energy consumption of a commercial building, located in Recife - PE, through the use of a thermoelectric unit (generator) that operates on natural gas, which will serve as an alternative source of energy in the energy matrix of the enterprise. The cost of electricity represents a considerable impact on the result in operating cost of any project, where every economy contributes to maintaining the viability of the business, which is the main reason for this analysis. The quantitative data analysis of energy consumption of
\end{abstract}

building Commercial studied, combined with knowledge of the hour-seasonal tariff system was essential to determine the project's feasibility. Moreover, it was determined the economic viability of the project, based on two financial parameters the return time (payback) and the net present value criterion (NPVC). The results show, that really is technically and economically feasible the implementation of this investment project, achieving a reduction of electricity consumption of approximately $20 \%$. m., equivalent to $R \$ 295,000.00$ less the amount paid monthly, and Commercial Building gained in diverse sources of electricity.

KEYWORDS: Financial Analysis, Thermoelectric, Reduced Power Consumption. 


\section{INTRODUÇÃO}

A energia elétrica é essencial para a subsistência dos seres humanos. Em processos produtivos são fundamentais no desenvolvimento de bens e serviços. Devido à importância da energia elétrica para a sobrevivência e desenvolvimento econômico, existe uma forte discussão sobre a melhor forma de utilização dos recursos naturais para produção de energia elétrica, pois a utilização exagerada desse recurso pode levar a sua escassez. Com isto, a energia elétrica deve ser utilizada com responsabilidade e com o melhor aproveitamento possível. No Brasil as hidrelétricas são as principais fontes de energia e representam aproximadamente $65 \%$ da energia elétrica gerada (ANEEL, 2012). Ao longo do tempo acreditava-se que esta seria a melhor forma de gerar energia. Entretanto, Outros tipos de fontes de energia alternativas estão ganhando força no Brasil, o que contribui para reduzir a dependência de uma única fonte e para o desenvolvimento da economia de forma sustentável no país (WANDERLEY e CAMPOS, 2013).

O custo com energia elétrica tem uma representatividade significativa nos custos operacionais dos edifícios comerciais e impactam diretamente nos resultados financeiros das empresas instaladas. Estes custos estão associados ao desperdício de energia devido ao uso incorreto, instalações inadequadas e a falta de aplicação de sistemas alternativos de geração de energia independente, que possibilitem a contratação de tarifas mais economicamente viáveis. Segundo SILVA (2009) o adequado entendimento da necessidade de inserção de fontes renováveis e alternativas na matriz brasileira passa por compreender que o modelo de geração de energia fundamentado em hidroelétricas com grandes reservatórios tende ao esgotamento. Do mesmo modo, CAVALCANTI e SILVA (2008) reforçaram o apresentado por SILVA, argumentando que com o aumento da demanda da energia elétrica devido ao crescimento do país, é necessário intensificar o estudo da diversificação da matriz energética, buscando o aproveitamento de recursos brasileiros e de novas tecnologias, como foi analisado em BRIZI et al., 2014, através do estudo de um sistema de cogeração de energia elétrica e térmica a partir da utilização de um único combustível como fonte de matéria prima.

Segundo ZANCAN et al., (2006), onde foi manifestado que a demanda de energia no Brasil se apresenta de forma totalmente irregular, originando períodos críticos de consumo, exigindo geração máxima, e em outros de baixa demanda, a geração torna-se ociosa. No Brasil, o principal período crítico de consumo, também chamado de horário de ponta corresponde ao intervalo entre as 18 e 21 horas. Por este motivo, cresce o número de empresas que, por razões de economia e segurança, optam pela contratação do fornecimento de energia elétrica pelo regime de tarifa hora-sazonal (tarifa azul e tarifa verde) e utilizam grupos geradores para o suprimento de energia elétrica nos horários de ponta, reduzindo seus custos com o consumo de energia elétrica, como pode ser verificado em SCHNEIDER e GHILARDI (2008). MACHADO (2010) realizou um estudo sobre gerenciamento de energia em uma planta industrial com o objetivo de reduzir os custos de operação, e abordou que se aumentando o número de fontes, que utilizam diferentes insumos, diminui-se as chances de falta de energia. Ainda, utilizando diferentes insumos, se ganha na flexibilidade de operação devido à possibilidade de escolher o insumo mais viável, de acordo com o cenário.

Diferentes trabalhos da literatura, tem demonstrado a análise econômica como ferramenta auxiliar da toma de decisões de investimento, e na busca de reduzir custos 
relacionados com o consumo de energia elétrica, através do reaproveitamento e utilização de outra fonte alternativa de energia elétrica (OLIVEIRA et al., 2000; ELEOTERO, 2008; EVELOY et al., 2014; PANTALEO et al., 2013; RIVAROLO et al., 2013; MANESH et al., 2014; WU et al., 2014).

SOMCHAROENWATTANA et al. (2011) apresentaram dois casos de alternativas de melhoria de desempenho. O primeiro é uma central de cogeração 52,5 MWe no Aeroporto de Suvarnabhumi, e a segunda é a usina de cogeração de 9,9 MW do complexo de edifícios do governo. Essa análise permitiu melhorar a eficiência energética, reduzir o consumo de energia primária, diminuir redução de emissões e proporcionou benefícios econômicos. No caso 1 , os resultados mostraram uma melhoria da eficiência global de $48 \%$ para $61 \%$, uma redução de $24 \%$ na utilização da energia primária, e uma diminuição de $27 \%$ na emissão de CO2. Devido a estes resultados, o lucro é aumentado para 24,80 milhões de \$/ano, com período de retorno de 4,77 anos. No caso 2, a aplicação de armazenamento de água refrigerada leva ao máximo de lucro de 2,63 milhões de \$/ano. Seguidamente, CAMPOS et al. (2011) realizaram uma análise de viabilidade em uma planta de pequena escala operada com um motor que opera com gás, sendo está a base da cogeração para sistemas residenciais que atuam segundo o regulamento espanhol. Um perfil de carga unitária térmica foi obtido considerando a demanda térmica de varias residências na Espanha, e foi utilizado para analisar o potencial de cogeração na faixa de pequena escala de potência (100-1000 kW). Foi verificado que o aumento do preço do gás natural aumenta a viabilidade de uma planta de cogeração enquanto decresce sua rentabilidade.

Por outro lado, TONIM (2009) observou a importância da escolha da melhor opção de tarifa de energia elétrica em uma fábrica de alimentos, e a forma de realizar a sua contratação, assim como a adoção de medidas que visam a obtenção da melhor eficiência energética, e DE SOUZA et al. (2010) apresentaram medidas de eficiência diversas distribuidoras de energia elétrica que operam no setor elétrico brasileiro. Essas medidas foram obtidas através de modelos de análise envoltória de dados e de fronteira estocástica, duas técnicas que podem mitigar a assimetria de informação e aperfeiçoar a habilidade do agente regulador, comparando os desempenhos das distribuidoras.

A importância de modelos econômicos que possam prever sistemas de produção seja energética ou manufatura, visando a criação de simuladores aproximados que permitam determinar o comportamento através da eficiência dos processos produtivos de diferentes áreas de produção (BRUNSTEIN e TOMIYA, 1995; PEREIRA e SILVEIRA NETO, 2012). Neste contexto, PIEKARSKI e CZIULIK (2009) apresentaram o desenvolvimento de um modelo para facilitar a tomada de decisão quanto ao emprego de sistemas de fixação desmontáveis, considerando aspectos econômicos envolvidos nos processos examinados. ALEM e MORABITO (2013) investigaram um problema combinado de planejamento da produção e corte de estoque comum em fábricas de móveis de pequeno porte, visando os custos de produção envolvidos no processo de manufatura e suas demandas dos produtos. O trabalho proporcionou propostas de modelos de otimização robusta que permitiram controlar o conservadorismo da solução de acordo com a atitude do responsável da decisão em relação ao risco. Da mesma forma, DA SILVA et al. (2013) propuseram um modelo de Programação por Metas Binária -Mista (MBGP), para otimizar o planejamento agregado da produção e da distribuição de uma empresa do setor sucroenergético, que incorpora a cogeração de energia, abordando os processos de produção de açúcar, de álcool, de melaço e derivados e da cogeração de energia. Finalmente, PASSOS e SOUZA (2013) apresentaram um método multicritério híbrido construído através da fusão entre o Método de 
Análise Hierárquica (AHP) e técnicas de Amortecimento Exponencial, aplicadas na previsão de séries temporais. As conclusões permitiram revelar a necessidade de levar em consideração os resultados obtidos em meses anteriores (histórico da empresa), mas dando menor importância a períodos mais distantes do tempo presente.

Este trabalho tem como objetivo reduzir o consumo de energia elétrica de um Edifício Comercial, localizado em Recife - PE, através da utilização de uma unidade termelétrica (grupo gerador) que opera com gás natural. A unidade termelétrica servirá como uma fonte alternativa de energia na matriz energética do empreendimento. A análise financeira do empreendimento determinou a viabilidade do investimento, encontrando uma redução na economia mensal de aproximadamente de $20 \%$ em energia elétrica.

\section{ANÁLISE ENERGÉTICA}

Para realizar a análise econômica do processo, foram estudados os diferentes modelos de tarifação existente, apresentados pela concessionária de energia elétrica.

\subsection{Modelos de tarifação de energia}

De acordo com a ANEEL - Agência Nacional de Energia Elétrica, a tarifa engloba todos os itens necessários para geração, transmissão, distribuição e comercialização da energia elétrica. Todos os consumidores pagam um valor relacionado à energia consumida no mês anterior, equivalente ao quilowatt-hora $(\mathrm{kWh})$ total do período, onde este valor é multiplicado pelo pela tarifa que é medida em reais por quilowatt-hora $(\mathrm{R} \$ / \mathrm{kWh})$. A estrutura tarifária corresponde ao conjunto de tarifas aplicáveis conforme nível de demanda de potência requerida, níveis de consumo e modalidade de fornecimento. As tarifas de demanda estão avaliadas em reais por quilowatt $(R \$ / k W)$ e as de consumo em reais por megawatt-hora ( $R \$ / M W h)$. Não são todos os consumidores que pagam tarifa de demanda. Dependerá da estrutura tarifária e da modalidade de fornecimento pela qual o consumidor está enquadrado. Estão subdivididas em dois grandes grupos de consumidores: Tarifas dos grupos A e B.

\subsection{Tarifas do grupo A}

Correspondem aos consumidores atendidos por tensão entre 2,3 e 230 quilovolts (kV), como mostrado na tabela 1, e são identificados por letras e algarismos que indicam a tensão de operação.

Tabela 1: Tarifas grupo A, Fonte: ANEEL (2012).

\begin{tabular}{|c|c|}
\hline Classe & Tensão \\
\hline A1 & $\geq 230 \mathrm{kV}$ \\
\hline A2 & $88-138 \mathrm{kV}$. \\
\hline A3 & $69 \mathrm{kV}$. \\
\hline A3a & $30-\mathbf{4 4} \mathrm{kV}$. \\
\hline A4 & $\mathbf{2 , 3}-\mathbf{2 5} \mathbf{~ k V}$. \\
\hline AS & Aplicação em sistema subterrâneo. \\
\hline
\end{tabular}

Tarifa convencional: Esta tarifa totalizará o consumo de energia elétrica acumulado no mês anterior e a demanda contratada pelo consumido, cujo valor permanece constante 
independente da hora do dia e do período do ano. Apresenta um valor para demanda de potência $(R \$ / k W)$ e outro para o consumo de energia ( $R \$ / M W h)$.

$$
\begin{aligned}
& P_{\text {consumo }}=\text { Tarif }_{\text {consumo }} \cdot \text { Consumo }_{\text {medido }} \quad \text { Equação (1) } \\
& P_{\text {demanda }}=\text { Tarif } a_{\text {demanda }} \cdot \text { Demanda } a_{\text {contratada }} \quad \text { Equação (2) }
\end{aligned}
$$

Tarifa hora-sazonal: Este modelo tarifário apresenta tarifas diferenciadas de demanda de potência e de consumo, conforme o horário de utilização do dia e dos períodos do ano. $O$ objetivo deste modelo tarifário é de racionalizar a utilização da energia conforme horário do dia e período do ano, motivando o consumidor a utilizar mais energia nos períodos e horários em que a energia estiver mais barata e desestimulando sua utilização em horários e períodos mais caros.

Tarifa hora-sazonal azul: Esta modalidade apresenta valores diferentes de tarifas para consumo de energia nos horários do dia e períodos do ano, bem como apresenta valores diferentes de demanda de potência de acordo com horários do dia. Sua aplicação é obrigatória para consumidores atendidos pelo sistema interligado que tenha tensão igual ou superior a 69 KV.

Demanda de potência $(\mathrm{RS} / \mathrm{kW})$ : Possui um valor para o horário de ponta e outro para fora de ponta.

$$
\begin{aligned}
P_{\text {demanda }}= & \text { Tarif } a_{\text {demanda_ponta }} \cdot \text { Demanda } \\
& + \text { Tarifontratada_ponta } a_{\text {contratada_fora }} \cdot \text { Demand } a_{\text {contratada_fora }}
\end{aligned}
$$

Consumo de energia (R\$/MWh): Possui um valor para horário de ponta em período úmido e outro para período seco. Possui um valor para horário fora de ponta para período úmido e outro para período seco.

$$
\begin{gathered}
P_{\text {consumo }}=\text { Tarifa } a_{\text {consumo_ponta }} \cdot \text { Consumo }_{\text {medido_ponta }}+\text { Tarifa } a_{\text {medido_fora }} \\
\cdot \text { Consumo }_{\text {medio_fora }}
\end{gathered}
$$

Tarifa hora-sazonal verde: Esta modalidade apresenta valores diferentes de tarifas para consumo de energia nos horários do dia e períodos do ano, mas apresenta uma única tarifa de demanda de potência para todos os horários do dia. Sua aplicação é obrigatória para consumidores atendidos pelo sistema interligado que tenha tensão inferior a $69 \mathrm{kV}$ e demanda igual ou inferior a $300 \mathrm{~kW}$, podendo o consumidor optar pela modalidade azul ou verde. Bem como os consumidores atendidos pelo sistema elétrico interligado que tenha tensão inferior a 69 $\mathrm{kV}$ e demanda maior que $300 \mathrm{~kW}$, estrutura da tarifa hora-sazonal verde.

Demanda de potência $(\mathrm{R} \$ \mathrm{~kW})$ : Valor único de tarifa.

$P_{\text {demanda }}=$ Tarif $a_{\text {demanda }} \cdot$ Demanda $a_{\text {contratada }}$

Equação (5) 


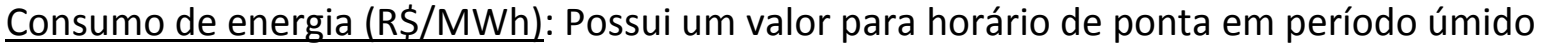
e outro para período seco. Possui um valor para horário fora de ponta para período úmido e outro para período seco.

\subsection{Tarifas do grupo $B$}

Estas tarifas são destinadas a consumidores que são atendidos com tensão inferior a 2,3 $\mathrm{kV}$, cujas classes de consumo dependem do tipo de empreendimento.

\subsection{Potência e consumo de energia}

\subsubsection{Potência elétrica}

Segundo CREDER (2007), para que seja possível realizar qualquer movimento ou produzir calor, luz, radiação e outras, dependemos da energia. Potência corresponde à aplicação desta energia na unidade de tempo, ou seja, é a energia aplicada por segundo para realizar qualquer uma das atividades relacionadas acima. Na eletricidade, potência corresponde ao produto da tensão com a corrente elétrica.

\subsubsection{Potência Aparente (S)}

Corresponde a potência total consumida, englobando a potência ativa mais a reativa.

$S=U \cdot I$

Equação (6)

Sendo:

$U=$ Voltagem $($ Volt $)$

$I=$ Corrente elétrica (Ampère)

\subsubsection{Potência Ativa (P)}

Corresponde a capacidade de um determinado circuito produzir trabalho em um determinado período de tempo.

$P=U \cdot I \cdot \operatorname{Cos} \emptyset$

Equação (7)

\subsubsection{Fator de Potência (FP)}

Representa a relação entre a potência ativa e a potência aparente (Total). Esta relação demonstra o quanto à energia está sendo usada adequadamente pela unidade consumidora, pois um fator de potência muito baixo gera perdas de energia.

$F P=\frac{P}{S}$

Equação (8)

$\cos \emptyset=F P$

Equação (9) 
$\emptyset$; corresponde ao ângulo de defasagem entre a tensão e a corrente.

\subsubsection{Potência Reativa (Q)}

Corresponde a potência necessária para gerar o campo elétrico e magnético que acionarão motores, geradores, transformadores, reatores e outros. Esta potência não gera trabalho e causam sobrecargas no sistema de transmissão. Por este motivo, concessionárias cobram multas por consumo excedente de energia reativa.

$Q=U \cdot I \cdot \sin \varnothing$

Equação (10)

\subsubsection{Consumo de Energia Elétrica}

Conforme CREDER (2007), o consumo de energia elétrica, representa o quanto de potência elétrica foi consumida em um determinado período de tempo.

\subsubsection{Consumo de Energia Ativa ( $\mathrm{kWh}$ )}

Consumo $=$ Potência Ativa $\cdot$ Tempo de Trabalho

Equação (11)

\subsubsection{Consumo de Energia Reativa (kVARh)}

Consumo $=$ Potência Reativa $\cdot$ Tempo de Trabalho

Equação (12)

\section{ANÁLISE ECONÔMICA}

Segundo FERREIRA (2000), investimento pode ser definido sob óticas diferentes. Em uma visão microeconômica ou economia de empresa, compreende-se investimento como toda aplicação de recursos econômicos no presente, objetivando a obtenção de lucros futuros. Numa visão macroeconômica ou de contabilidade nacional, o conceito de investimento está fundamentado no aumento líquido ocorrido na estrutura do capital, detectado nos setores da economia. A avaliação prévia de qualquer investimento empresarial é fundamental para que seja possível determinar a viabilidade econômica da aplicação de recursos financeiros que poderiam ser direcionados para outras situações que trouxesse melhores resultados. Os ativos de investimentos empresariais possuem longo período de vida útil, onde tudo que é previsto deve atender as condições de fluxo de caixa da organização.

\subsection{Tempo de retorno (Payback)}

Representa a relação entre o valor do investimento e o fluxo de caixa do projeto. Indica em quanto tempo ocorrerá a recuperação do investimento realizado no projeto. Tem como pontos fracos não considerar o valor do dinheiro em função do tempo, não considerar os fluxos de caixa após a recuperação do capital e não poder ser aplicado quando o fluxo de caixa alterna o sinal de positivo para negativo ou vice-versa. 
tempo $_{\text {retorno }}=$ Custo Inicial $($ Investimento $) /$ Lucro Operacional

Equação (13)

\subsection{Critério do valor presente líquido (CVPL)}

Este critério se baseia na atualização do fluxo de caixa representativos de receitas ou faturamentos, custos ou despesas e lucros operacionais, para determinada previsão de planejamento, sendo empregado como taxa de desconto a taxa mínima de atratividade (TMA). Seu objetivo econômico é que o lucro do projeto de investimento supere o ganho ou juros de operações do mercado financeiro, ou de outras operações com taxa de retorno já definida. A continuação é apresentado o modelo matemático para se determinar o VPL do projeto.

$V P L\left(i_{m}\right)=\sum_{t=0}^{n} \frac{R_{t}-C_{t}}{\left(1+i_{m}\right)^{t}}-I_{0}$

Equação (14)

$i_{m}$ - Taxa mínima de atratividade

$R_{t}-$ Receita acumulada no período

$C_{t}$ - Custo acumulado no período

$t$-Tempo de análise

$I_{0}$ - Investimento inicial

\section{ANÁLISE E DISCUSSÃO DOS RESULTADOS}

O empreendimento objeto do estudo possui mais de 450 lojas instaladas e um consumo mensal de energia aproximadamente $3.560 .000 \mathrm{KWh}$, o que equivale a aproximadamente 24.000 residências com um consumo médio de $150 \mathrm{KWh}$. Através de um investimento empresarial em uma termelétrica a gás natural, é possível obter economia financeira vantajosa para o empreendedor. Inicialmente foi necessário o levantamento dos dados de consumo de energia do Edifício Comercial para analisar as características e particularidades necessárias para que fosse possível determinar tecnicamente as melhores opções de investimento. Para o estudo de caso foram observados os parâmetros necessários, seguindo as etapas relacionadas abaixo para que fosse possível tomar a decisão de investimento.

- Análise de consumo de energia (Ponta e Fora de Ponta).

- Análise de demanda de potência (Ponta e Fora de Ponta).

- Definição do melhor modelo tarifário para o caso.

- Determinação do investimento necessário.

- Analise de retorno do investimento.

O Edifício Comercial em questão estava enquadrado inicialmente no modelo de tarifa hora-sazonal azul, onde o objetivo foi reduzir o custo com energia elétrica através da utilização de uma termelétrica a gás natural no horário de ponta.

\subsection{Primeira Etapa - Análise do consumo de energia do Edifício Comercial}

A análise do consumo nas condições ponta e fora de ponta do Edifício Comercial foi fundamental para verificar o perfil sazonal do cliente. Foram tomadas como parâmetros de 
análise três contas de energia elétrica do empreendimento, e seus resultados mostrados na figura 1.

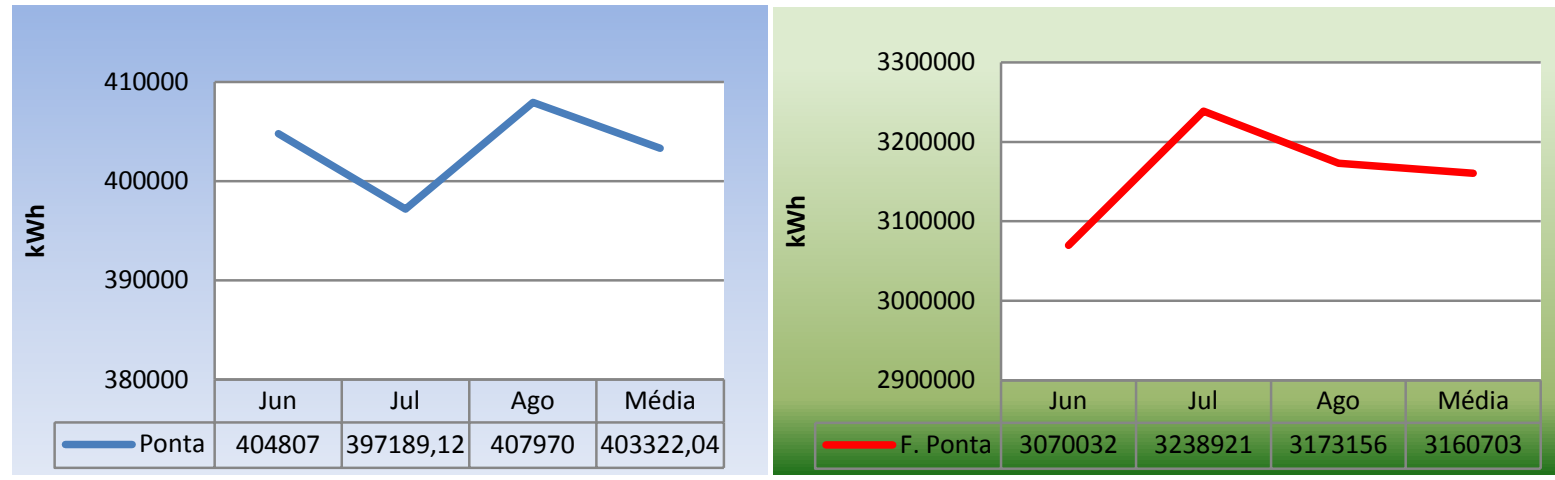

Figura 1 - Gráfico consumo de energia na ponta do Edifício Comercial.

Pode-se observar na figura 1, um consumo médio de $403.322 \mathrm{kWh}$ de energia na ponta, sem grandes variações sazonais observadas (Máximo 2,5\%). Para o período de fora de ponta, encontrou-se um consumo médio de 3.160 .703 kWh também sem variações consideráveis. De acordo com o Edifício Comercial não existem variações consideráveis de consumo entre os períodos sazonal úmido e seco

\subsection{Segunda Etapa - Análise da demanda de potência do edifício comercial}

A análise da demanda, figura 2, contribui para ajudar na determinação no modelo de tarifação e também da potência elétrica do sistema termelétrico a ser instalado. Nos gráficos é observado uma potência máxima registrada na ponta de $5839 \mathrm{~kW}$ e fora de ponta de $8302 \mathrm{~kW}$. Com isto, a demanda contratada pelo Edifício Comercial na ponta ficou em $6000 \mathrm{~kW}$ e fora de ponta $8300 \mathrm{~kW}$.
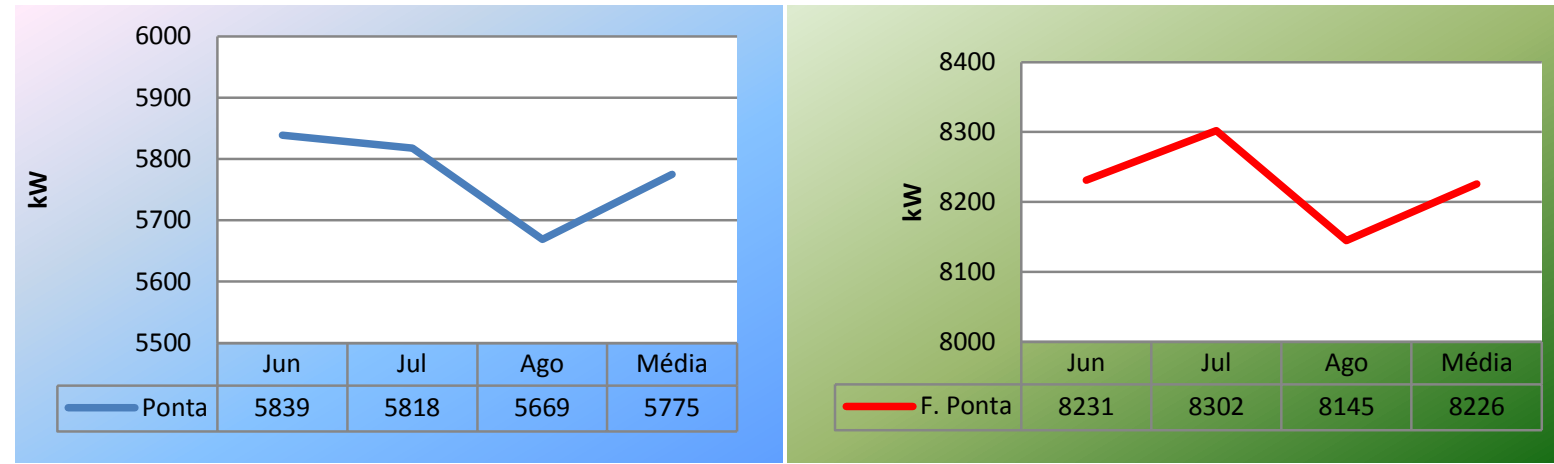

Figura 2 - Gráficos de demanda na ponta e fora de ponta do Edifício Comercial.

\subsection{Terceira etapa - Definição do melhor modelo tarifário para o caso}

Para definição do modelo tarifário fez-se necessário avaliar através de simulações nos modelos de tarifação possíveis para o consumidor, utilizando resultados obtidos no histórico de consumo que foram retirados das últimas contas de energia do Edifício Comercial. Para tal, foi avaliada a condição da melhor tarifa antes da instalação de uma termelétrica e depois da instalação de uma termelétrica para reduzir o consumo na ponta. A tabela 2 mostra a descrição da tarifa hora-sazonal azul e verde aplicada aos consumidores do subgrupo A4 da Companhia 
Energética de Pernambuco - CELPE, estabelecida pela ANEEL de acordo com a resolução homologatória no 1.283, de 24 de Abril de 2012.

Tabela 2 - Tarifas hora-sazonais azul e verde subgrupo A4 da CELPE

\begin{tabular}{|c|c|c|c|c|c|c|c|}
\hline \multicolumn{8}{|c|}{ SUBGRUPO A4 } \\
\hline \multicolumn{8}{|c|}{ AZUL } \\
\hline \multirow{2}{*}{\multicolumn{2}{|c|}{ Demanda $(\mathrm{R} \$ / \mathrm{kW})$}} & \multicolumn{2}{|c|}{ Úmido } & \multirow{2}{*}{\multicolumn{2}{|c|}{ Demanda $(\mathrm{R} \$ / \mathrm{kW})$}} & \multicolumn{2}{|c|}{ Seco } \\
\hline & & \multicolumn{2}{|c|}{ Consumo (R\$/kWh) } & & & \multicolumn{2}{|c|}{\begin{tabular}{|l} 
Consumo (R\$/kWh) \\
\end{tabular}} \\
\hline Ponta & F. Ponta & Ponta & F. Ponta & Ponta & F. Ponta & Ponta & F. Ponta \\
\hline 72,0022 & 23,01688 & 0,3444 & 0,2095 & 72,0022 & 23,0168 & 0,3827 & 0,2317 \\
\hline \multicolumn{8}{|c|}{ VERDE } \\
\hline \multirow{2}{*}{\multicolumn{2}{|c|}{ Demanda $(\mathrm{R} \$ / \mathrm{kW})$}} & Úm & & \multirow{2}{*}{\multicolumn{2}{|c|}{ Demanda $(\mathrm{R} \$ / \mathrm{kW})$}} & \multicolumn{2}{|c|}{ Seco } \\
\hline & & \multicolumn{2}{|c|}{ Consumo (R\$/kWh) } & & & \multicolumn{2}{|c|}{ Consumo $(\mathrm{R} \$ / \mathrm{kWh})$} \\
\hline Ponta & F. Ponta & Ponta & F. Ponta & Ponta & F. Ponta & Ponta & F. Ponta \\
\hline \multicolumn{2}{|c|}{23,0168} & 2,01624 & 0,20955 & \multicolumn{2}{|c|}{23,0168} & 2,0545 & 0,2317 \\
\hline
\end{tabular}

\subsubsection{Antes e depois da aplicação de uma termelétrica}

Foi avaliado graficamente o comportamento da conta de energia elétrica, sabendo que o Edifício Comercial estava inicialmente na tarifa azul. Para a simulação do sistema considerando uma termelétrica, foi necessário considerar, segundo o fabricante, uma disponibilidade mínima de $95 \%$ da termelétrica, devido às paradas para manutenções preventivas e corretivas. Além disto, também foi considerada uma sobra residual para concessionária de $1000 \mathrm{kWh} /$ dia no horário de ponta, pois os geradores trabalhariam em paralelo com a concessionária. Os resultados são mostrados na figura $3 a$ e ab, respectivamente.
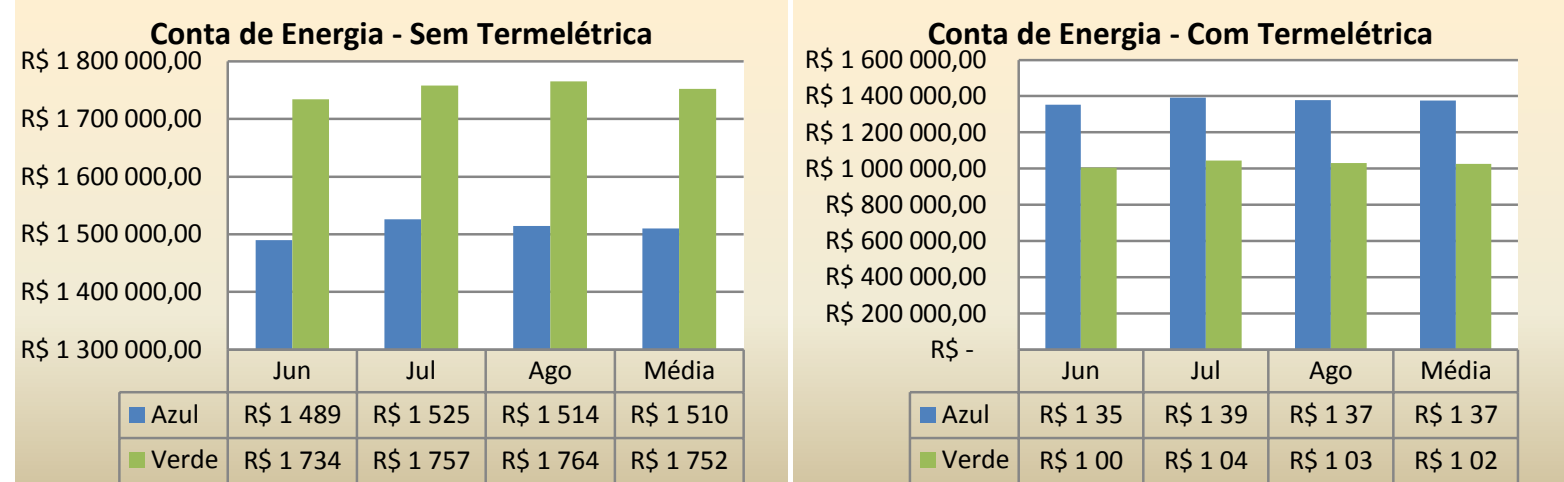

Figura 3 - a. Conta de energia do Edifício com termelétrica na azul e verde. b. Conta de energia do Edifício Comercial sem termelétrica.

$\mathrm{Na}$ condição sem termelétrica, figura 3a, é possível constatar que com a tarifa azul, o Edifício tinha uma redução média aproximada de $\mathrm{R} \$ 240.000,00 /$ mês em relação à tarifa verde, representando aproximadamente $14 \%$ de economia. Com isto, é possível concluir que na condição sem termelétrica o Edifício estava corretamente colocado no modelo de tarifação horasazonal azul. Na condição com termelétrica, figura $3 \mathrm{~b}$, o resultado demonstra que na tarifa verde o custo com energia ficou aproximadamente $\mathrm{R} \$ 350.000,00 /$ Mês menor que na tarifa azul. 
Obviamente que nesta analise inicial não estão sendo considerados os custos com combustível e manutenção dos geradores de energia.

Segundo os resultados obtidos com a aplicação da termelétrica, foi realizada uma análise, considerado o funcionamento desta termelétrica no horário de ponta, tanto na tarifa azul quanto na verde, mostrada na figura 4.

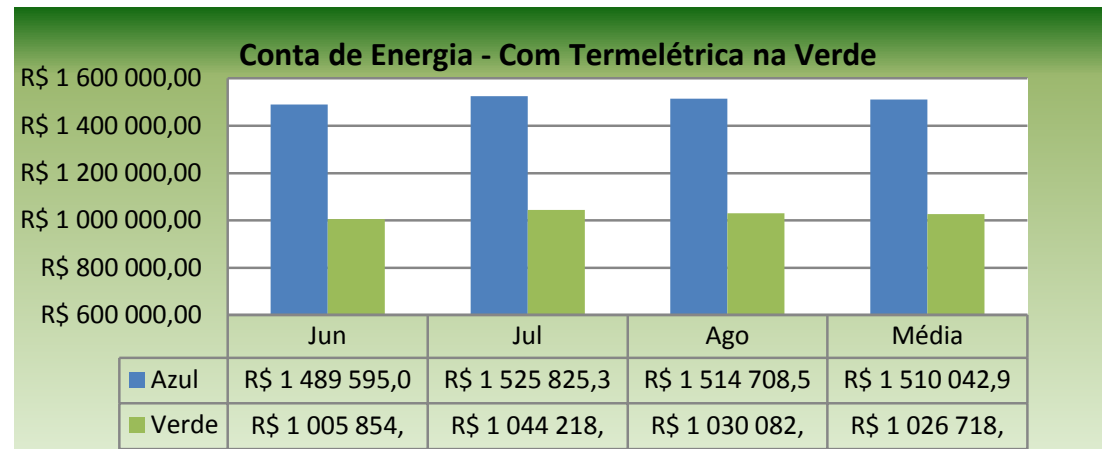

Figura 4 - Conta de energia do Edifício com termelétrica.

Conforme figura 4, após a instalação da termelétrica a conta de energia caiu aproximadamente $\mathrm{R} \$ 480.000,00 /$ Mês, equivalente a $32 \%$ de redução. A tabela 4 mostra o consumo de energia com a termoelétrica no período seco.

\subsection{Quarta etapa - Determinação do investimento necessário}

Após analisar consumo, demanda e melhor modelo tarifário para as condições possíveis, chegou a hora de definir os equipamentos necessários de uma termelétrica a gás natural que atendesse as condições técnicas para o funcionamento fundamentalmente em horário de ponta para redução de consumo. O Edifício Comercial tem uma demanda máxima registrada na ponta de aproximadamente $5.840 \mathrm{~kW}$. Para atender a demanda o Edifício Comercial optou-se por instalar uma termelétrica de $6.000 \mathrm{~kW}$ equipada com sistema de transferência em rampa para que não houvesse interrupção no fornecimento de energia durante a transição entre as fontes de energia. Para operação em horário fora de ponta, a potência de 6000 kW não é suficiente para atender em $100 \%$ uma situação de emergência (Falta de energia). Nesta condição o empreendimento optou por priorizar cargas críticas com os geradores e deixar as cargas não críticas desligadas até o restabelecimento da concessionária, reduzindo assim o seu investimento em equipamentos. Para tal, o Edifício Comercial optou pela aquisição de três geradores de 2.000 kW fornecidos pela Cummins Power Generation, com sistema de transferência automático. Os geradores são do modelo QSV91 são mostrados na figura 5 .
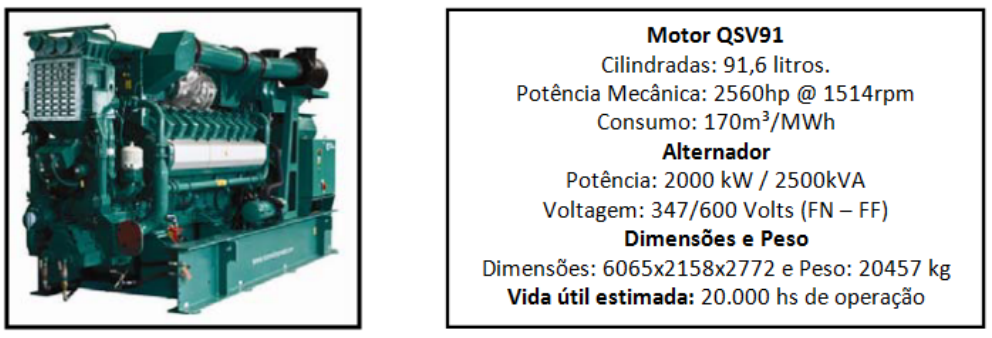

Figura 5 - Especificações técnicas do grupo gerador Cummins. (Fonte: Cummins, 2012) 
O investimento total para instalação do sistema termelétrico ficou em aproximadamente $\mathrm{R} \$$ 8.200.000,00. O Edifício Comercial contratou o fornecimento de gás natural encanado, recebendo diretamente do distribuidor de gás associado à Abegás - Associação Brasileira de Empresas Distribuidoras de Gás Canalizado. A concessionária de gás local fornece o gás a um custo de $\mathrm{R} \$ 1,3109 / \mathrm{m}^{3}$ na faixa 1 (Primeiros $1000 \mathrm{~m}^{3}$ ) e $\mathrm{R} \$ 1,2877$ na faixa 2 (Depois dos $1000 \mathrm{~m}^{3}$ ) fornecidos. Considerando que o sistema tem uma demanda média diária de potência na ponta de $5775 \mathrm{~kW}(5,77 \mathrm{MW})$, trabalhando 3 horas por dia e que o consumo de um gerador com motor de combustão interna a gás natural fica em aproximadamente $270 \mathrm{~m}^{3} / \mathrm{MWh}$, encontramos um consumo médio mensal aproximado de $95572 \mathrm{~m}^{3}$ a um custo mensal médio de $\mathrm{R} \$ 123.600,00$.

\subsection{Quinta etapa - Analise do Retorno do Investimento}

Para avaliar o retorno deste investimento foram utilizados os métodos de análise do tempo de retorno (Payback) e Critério do valor presente líquido (CVPL), mostrado na figura 6. Para ambos os casos foi necessário saber qual o lucro operacional do projeto. Foi Considerado como lucro operacional o resultado obtido pela diferença entre as receitas acumuladas pela redução do consumo com a utilização de uma termelétrica a gás, e as despesas para manter o sistema operacional no período.

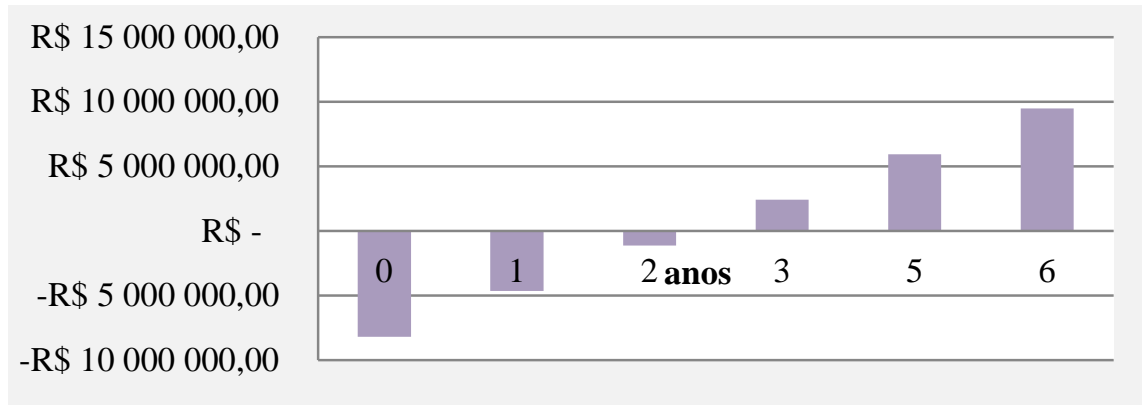

Figura 6 - Tempo de retorno do investimento do Edifício Comercial

Assim também, foi considerada como receita a redução do consumo que ficou em aproximadamente $\mathrm{R} \$ 480.000,00 /$ Mês. Para as despesas foi necessário avaliar o custo com combustível que ficou na ordem de aproximados $\mathrm{R} \$ 123.600,00 /$ Mês e o custo com manutenção da termelétrica ficou em aproximados $R \$ 62.000,00 /$ Mês (Contrato de operação, manutenção preventiva e corretiva). Com isto, as despesas mensais para manter a operação da usina ficaram em aproximadamente $\mathrm{R} \$ 185.600,00$. Isto nos deu um lucro operacional de $\mathbf{R} \$ \mathbf{2 9 5 . 0 0 0 , 0 0}$ a.m. ( $\mathbf{R} \mathbf{\$} 3.540 .000,00$ a.a.), o que corresponde a uma redução final de aproximadamente $\mathbf{2 0 \%}$ no custo com energia. Por este método foi possível concluir que com aproximadamente 2,3 anos o projeto seria totalmente pago pela economia mensal.

Contudo, conforme relatado anteriormente este método não avalia o valor do dinheiro no tempo. Para tal, foi analisado o critério do valor presente líquido (CVPL). Nesta condição, mostrado na figura 7, considerando o tempo do projeto em função da vida útil dos equipamentos (20.000h), o que equivale a aproximadamente 25 anos de operação, e utilizando uma taxa mínima de atratividade de 7,25\% a.a. (Taxa Seliq Banco Central, Out/2012), foi encontrado o VPL de $\mathrm{R} \$ 32.141 .005,73$. 


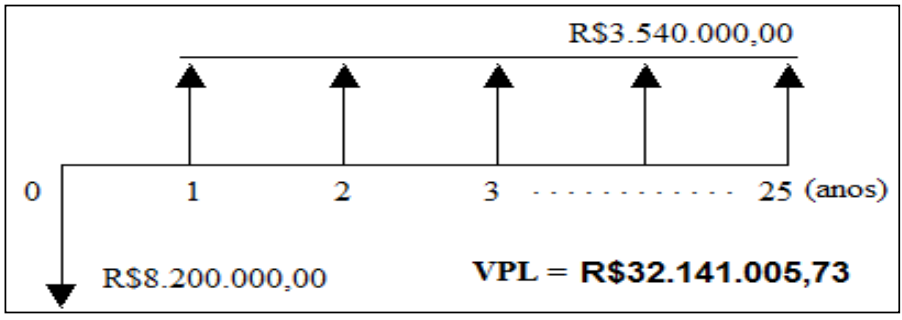

Figura 7 - Gráfico VPL do Edifício Comercial.

Portanto, significa que o investimento foi economicamente viável, pois trazendo o valor do dinheiro para o momento atual, obtivemos um resultado bastante positivo.

\section{CONCLUSÃO}

Pode-se verificar a viabilidade da instalação de uma termelétrica a gás natural para reduzir o custo com energia no horário de ponta em um Edifício Comercial, localizada em Recife PE. Entretanto, foi necessária a realização de análises quantitativas de consumo de energia elétrica do empreendimento, observando as variações sazonais do período seco e úmido, e horário do dia, ponta e fora de ponta. Além disto, a análise do melhor modelo de tarifação para o caso demonstrou que em aplicações com termelétrica para consumidor que está dentro do grupo A4, a tarifa hora-sazonal verde é a melhor opção para obtenção do melhor resultado econômico.

A análise financeira do empreendimento foi fundamental para determinar a viabilidade do investimento, onde foi obtida uma economia mensal de aproximadamente $\mathrm{R} \$ 295.000,00$, equivalente a $20 \%$ a menos no custo com energia elétrica. Foram utilizados métodos reconhecidamente confiáveis para contribuir com a decisão final.

O tempo de retorno do investimento (payback) do projeto demonstrou que num tempo curto de 2,3 anos o investimento seria pago pela economia com energia elétrica do período e o critério do valor presente líquido contribuiu para que fosse possível avaliar o valor do dinheiro no tempo presente, onde encontramos um valor aproximado de $\mathrm{R} \$ 32.000 .000,00$ para o período de vida útil dos equipamentos.

Finalmente, a diversificação da matriz energética em um Edifício Comercial contribuiu também para transmitir tranquilidade aos investidores, pois além de ajudar a reduzir o custo com energia elétrica, foi possível sua utilização em condições emergenciais para manter o empreendimento operacional, principalmente em momentos de desconfiança com a qualidade e capacidade de distribuição de energia dos principais fornecedores nacionais.

\section{REFERÊNCIAS BIBLIOGRÁFICAS}

1. ALEM, D., MORABITO, R. O problema combinado de planejamento da produção e corte de estoque sob incertezas: Aplicação em fábricas de móveis de pequeno porte. Gestão \& Produção, v. 20, n. 1, p. 113 - 133, 2013.

2. ANEEL - Agência Nacional de Energia Elétrica,MatrizdeEnergiaElétricadoBrasil. Disponível em: <http://www.aneel.gov.br/aplicacoes/capacidadebrasil/Operacao

3. CapacidadeBrasil.asp> Acesso em 07 out 2012. 
4. BRIZI, F., SILVEIRA, J., DESIDERI, U., REIS, J., TUNA, C. E., LAMAS, W. Energetic and economic analysis of a Brazilian compact cogeneration system: Comparison between natural gas and biogas. Renewable and Sustainable Energy Reviews, v. 38, n. 1, p. 193-211, 2014.

5. BRUNSTEIN, I. TOMIYA, E. H. O modelo econômico de Empresa Sucroalcooleira. Gestão \& Produção, v. 2, n. 3, p. 264 - 280, 1995.

6. CUMMINGS - Cummings Power Generation Brasil. Disponível em: <http://www.cummins power.com.br/pdf/gas/leanburn/s-1463.pdf> Acesso em 07 de set 2012.

7. CAMPOS A. C., ERKOREKA, A., ESCUDERO, K. M., SALA, J. M. Feasibility of small-scale gas engine-based residential cogeneration in Spain. Energy Policy, v. 39, n. 6, p. 3813 - 3821, 2011.

8. CAVALCANTI, A. L. M., SILVA, L. D. Estudo da expansão do setor elétrico brasileiro com geração termelétrica à gás natural. Trabalho de Conclusão de Curso. Departamento de Engenharia Elétrica, Universidade de Brasília, Dez. 2008.

9. CREDER, Hélio. Instalações Elétricas, 2007. Editora LTC 15a Edição.

10. DA SILVA, A. F., MARINS, F. A. S., MONTEVECHI, J. A. B. Aplicação de programação por metas binária - mista em uma empresa do setor sucroenergético. Gestão \& Produção, v. 20, n. 2, p. 321 - 336, 2013.

11. DE ARAUJO, M. V., SOUZA, R. C., PESSANHA, J. F. M. Custos operacionais eficientes das distribuidoras de energia elétrica: um estudo comparativo dos modelos DEA e SFA. Gestão \& Produção, v. 17, n. 4, p. 653 - 667, 2010.

12. ElEOTERO, B. C. Redução do Custo com Energia Elétrica em Sistemas de Bombeamento Estudo de Caso Sistema de Abastecimento de Água de Capinzal/Ouro - (SC). Trabalho de Conclusão de Curso. Departamento de Engenharia Sanitária e Ambiental, Universidade Federal de Santa Catarina, Dez, 2008.

13. EVELOY $V$, RODGERS $P$, POPLI S. Trigeneration scheme for a natural gas liquids extraction plant in the Middle East. Energy Conversion and Management, v. 78, n. 1, p. 204-218, 2014.

14. FERREIRA, R. G. Matemática Financeira Aplicada, Mercado de Capitais, Administração Financeira e Engenharia Econômica. Editora Atlas, 7ạ Edição (2010).

15. KONG XQ, WANG RZ, LI Y, HUANG XH. Optimal operation of a micro-combined cooling, heating and power system driven by a gas engine. Energy Conversion and Management, v. 50, n. 3,p. 530-538, 2009.

16. LIANG $\mathrm{Y}$, SHU G, TIAN H, LIANG X, WEI H, LIU L. Analysis of an electricity-cooling cogeneration system based on RC-ARS combined cycle aboard ship. Energy Conversion and Management, v. 76, n. 1,p. 1053-1060, 2013.

17. MACHADO, A. R. A. Gerenciamento de Energia em Planta Industrial Visando a Redução dos Custos de Operação. Trabalho de Conclusão de Curso. Departamento de Engenharia Elétrica, Universidade Federal de Rio de Janeiro, 2010.

18. MANESH MHK, NAVID P, BAGHESTANI M, ABADI SK, ROSEN ME, BLANCO AM, AMIDPOUR M. Exergoeconomic and exergoenvironmental evaluation of the coupling of a gas fired steam power plant with a total site utility system. Energy Conversion and Management, v. 77, n. 1, p. 469-483, 2014. 
19. OLIVEIRA A. D., SCOLFORO, J. R. S., SILVEIRA, V. P. Análise Econômica De Um Sistema AgroSilvo-Pastoril Com Eucalipto Implantado Em Região De Cerrado. Revista Ciência Florestal. Paraná, v. 10, p. 1- 9, 2000.

20. PANTALEO AM, CAMPOREALE SM, SHAH N. Thermo-economic assessment of externally fired micro-gas turbine fired by natural gas and biomass: Applications in Italy. Energy Conversion and Management, v. 75, n. 1, p. 202-213, 2013.

21. PASSOS, A. C., SOUZA, R. C. Defining a quality index for electric power utilities using multiple criteria decision support and time series analysis. Gestão \& Produção, v. 20, n. 1, p. 1 - 12, 2013.

22. PEREIRA, A. C., SILVEIRA NETO, O. Viabilidade econômica de projetos e aspectos particulares em empreendimentos tipo projeto finance aplicados a investimentos em infraestrutura de transportes. HOLOS, v. 6, n. 1, p. 203-219, 2012.

23. PIEKARSKI, J. C. B., CZIULIK, C. Modelo para avaliação econômica comparativa entre alternativas de sistemas de fixação com elementos roscados. Gestão \& Produção, v. 16, n. 3, p. 450 - 465, 2009.

24. RIVAROLO M, GRECO A, MASSARDO AF. Thermo-economic optimization of the impact of renewable generators on poly-generation smart-grids including hot thermal storage. Energy Conversion and Management, v. 65, n. 1, p. 75-83, 2013.

25. SCHNEIDER, V. R. GHILARDI, W. J. Eficientização Energética: Uma Maneira de Reduzir os Custos com Energia Elétrica. Revista Eletrônica de Contabilidade, v. 5, p. 1- 11, Jan. 2008.

26. SILVA, W. D. A Cogeração de Energia Elétrica a Partir da Biomassa Inserida na Matriz Energética Brasileira. Trabalho de Conclusão de Curso. Departamento de Engenharia Elétrica, Universidade São Paulo, Set, 2009.

27. SOMCHAROENWATTANA, W., MENKEA, C. KAMOLPUSB, D., GVOZDENACC, D. Study of operational parameters improvement of natural-gas cogeneration plant in public buildings in Thailand. Energy and Buildings, v. 43, n. 4, p. 925-934, 2011.

28. TONIM, G. A Gestão da energia elétrica na indústria - Seu suprimento e uso eficiente. Dissertação de Mestrado. Departamento de Engenharia de Energia e Automação Elétrica, Universidade de São Paulo, Out. 2009.

29. WANDERLEY, A. C. F., CAMPOS, A. L. P. S. Perspectivas de inserção da energia solar fotovoltaica na geração de energia elétrica no rio grande do norte. HOLOS, v. 3, n. 1, p. 3-14, 2013.

30. WU, W., WANG, B., SHI, W., LI, X. Techno-economic analysis of air source absorption heat pump: Improving economy from a design perspective. Energy and Building, v. 81, n. 1, p. 200- 210, 2014.

31. ZANCAN, M. D., CASTELLANELLI, C., RUPPENTHAL, J. E., HOFFMANN, R. Utilização de fontes alternativas de energia em supermercados de médio e grande porte visando a autosuficiência energética no horário de ponta e a redução de impactos ambientais. XIII SIMPEP Bauru, SP, 2006. 